\title{
Erosion of a cohesive granular material by an impinging turbulent jet
}

\author{
Mingze Gong ${ }^{1}$, Sivar Azadi ${ }^{1}$, Adrien Gans ${ }^{2}$, Philippe Gondret $^{3}$, and Alban Sauret ${ }^{1, *}$ \\ ${ }^{1}$ Department of Mechanical Engineering, University of California, Santa Barbara, USA \\ ${ }^{2}$ Aix Marseille University, CNRS, IUSTI, Marseille, France \\ ${ }^{3}$ University Paris-Saclay, CNRS, Laboratoire FAST, Orsay, France
}

\begin{abstract}
The erosion of a cohesive soil by an impinging turbulent jet is observed, for instance, during the landing of a spacecraft or involved in the so-called jet erosion test. To provide a quantitative understanding of this situation for cohesive soils, we perform experiments using a model cohesion controlled granular material that allows us to finely tune the cohesion between particles while keeping the other properties constant. We investigate the response of this cohesive granular bed when subjected to an impinging normal turbulent jet. We characterize experimentally the effects of the cohesion on the erosion threshold and the development of the crater. We demonstrate that the results can be rationalized by introducing a cohesive Shields number that accounts for the inter-particles cohesion force. The results of our experiments highlight the crucial role of cohesion in erosion processes.
\end{abstract}

\section{Introduction}

The erosion of soils by localized flow and jet occurs in various civil, environmental, and aerospace engineering situations. For example, such a situation is encountered when a rocket takes off or lands due to the turbulent jet used for propulsion [1]. This situation is also encountered during measurements of soil cohesion before the construction of civil structures [2] as erosion can later leads to the collapse of bridge piers [3]. The erosion by a jet is also of great interest in clean-up processes, such as at nuclear sites that use this process to rid reactor surfaces of harmful particles [4].

In the case of a non-cohesive medium, the erosion of the grains by a fluid flow is controlled by the balance of the gravity force acting on a grain, which tends to prevent erosion, and the stress exerted by the flow, which induces the erosion. The initiation of the motion of a grain of diameter $d$ is governed by the ratio of the fluid shear stress, $\tau_{f} d^{2}$, and its apparent weight $\left(\rho_{g}-\rho_{f}\right) g d^{3}$, where $\rho_{g}$ and $\rho_{f}$ are the density of the grain and the fluid, respectively. The ratio of these two forces leads to the Shields number: $\mathrm{Sh}=\tau_{f} /\left[\left(\rho_{g}-\rho_{f}\right) g d\right]$. Below a threshold value of Sh no erosion occurs, whereas above this threshold, particles are eroded and transported by the flow. The threshold value of Sh depends on the nature of the flow, laminar or turbulent, but also on the particle Reynolds number, $R_{p}=u d / v$, where $u$ is the characteristic velocity of the flow and $v$ is the kinematic viscosity of the fluid.

A relevant situation in many applications consists of impacting a jet oriented perpendicularly to the surface. For instance, such a situation has been studied to characterize the Jet Erosion Test [2], where the depth eroded over

*e-mail: asauret@ucsb.edu

A video is available at https://doi.org/10.48448/d34p-wj46 time is measured. Using empirical law of erosion, it is then possible to obtain information on the erodibility of the sediment layer. To refine the description of this situation, different model studies have considered the erosion of non-cohesive granular media in air or underwater [5-7] and have shown that the erosion threshold can be predicted using a free jet model, taking into account the position of the virtual origin of the jet.

The influence of cohesion between the particles remains less understood. However, model cohesive bonds between grains remain more challenging to create. A recent study has considered solid, cohesive bonds between millimetric grains sizes [8]. However, with solid bonds, once the particles are eroded, the solid bonds break irreversibly, and the grains are not cohesive anymore. Here, we address the situation of cohesive grains, where the bonds can be formed again after the erosion occurs, similarly to the behavior of dust particles. To do so, we rely on an experimental method recently developed to create a cohesion-controlled granular material [9], and we consider experimentally the erosion of a flat granular bed made of cohesive spherical beads by an impacting turbulent jet.

\section{Experimental methods}

\subsection{Experimental setup}

The experimental system used to characterize the erosion threshold of a cohesive granular medium is shown in Fig. 1. In summary, the granular medium is placed in a cylindrical metal container of diameter $20.3 \mathrm{~cm}$ and height $5.1 \mathrm{~cm}$. The granular medium fills the container and exhibits a flat surface. A nozzle with an internal diameter of 


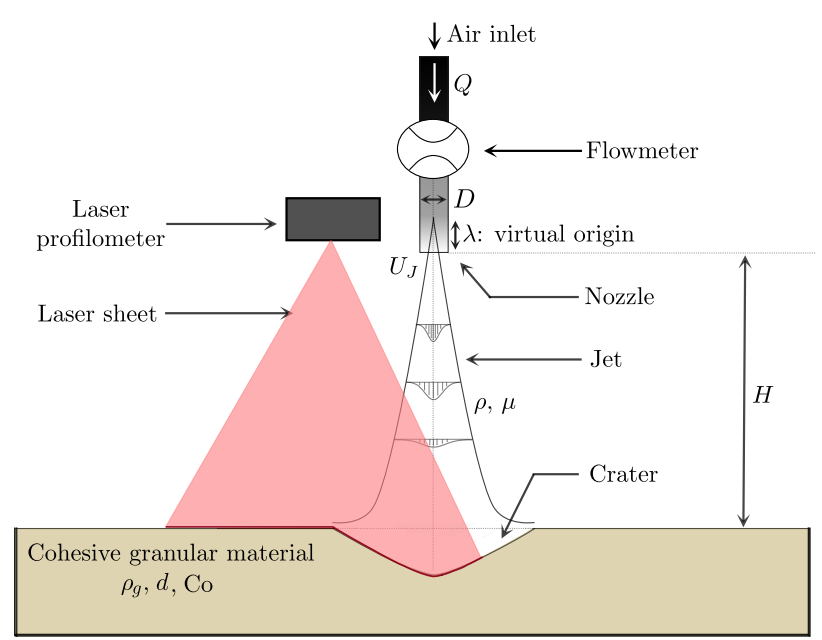

Figure 1. Schematic of the experimental setup. A jet exiting the nozzle of diameter $d$ at the mean velocity $U_{J}$ impacts the granular bed placed at a distance $H$.

$D=4.8 \mathrm{~mm}$ and a length of $50.8 \mathrm{~mm}$ is centered at the vertical of the granular bed. This nozzle is connected to compressed air via a PVC tube. The experiments are carried out at room temperature $\left(23.5^{\circ} \mathrm{C} \pm 1{ }^{\circ} \mathrm{C}\right)$ and in this condition the air has a density $\rho_{a}=1.19 \mathrm{~kg} . \mathrm{m}^{-3}$ and a dynamic viscosity $\eta=1.50 \times 10^{-5} \mathrm{~Pa}$.s. The distance between the outlet of the nozzle outlet and the surface of the granular bed is varied in the range $1 \mathrm{~cm}<H<22 \mathrm{~cm}$. The tubing is connected to a valve, which allows us to adjust the flow rate $Q_{J}$ of the jet, measured with a flowmeter. The flow rate is varied in the range $10^{-5} \mathrm{~m}^{3} . \mathrm{s}^{-1}<Q<10^{-3} \mathrm{~m}^{3} . \mathrm{s}^{-1}$, leading to an average velocity of the jet at the outlet of the nozzle of $U_{J}=4 Q_{J} /\left(\pi D^{2}\right) \in[0,50] \mathrm{m} / \mathrm{s}$, measured with an accuracy of $\pm 2 \%$.

\subsection{Cohesion-controlled granular material}

Adding cohesion to the particles is achieved using the method recently developed by Gans et al. [9]. In summary, spherical glass beads of density $\rho_{g}=2500 \mathrm{~kg} \cdot \mathrm{m}^{3}$ and diameter $d=800 \mu \mathrm{m}$ are coated by polyborosiloxane (PBS) made from an -OH terminated polydimethylsiloxane (PDMS) cross-linked with boric acid $\left(\mathrm{H}_{3} \mathrm{BO}_{3}\right)$. The coating thickness $b$ controls the strength of the cohesive force between two particles, which is captured by the relation

$$
F_{c}=\frac{3}{2} \pi \gamma d\left(1-e^{-b / B}\right)
$$

where the two fitting parameters are the characteristic length $B \approx 230 \mathrm{~nm}$ and the interfacial tension of the PBS $\gamma=24 \mathrm{mN} \cdot \mathrm{m}^{-1}$. Here, the coating thickness $b$ is varied between $0 \mathrm{~nm}$ (cohesionless grains) and $b=300 \mathrm{~nm}$. The resulting macroscopic cohesive stress of the material, $\tau_{c}$, is related to the microscopic inter-particle force $F_{c}$ through:

$$
\tau_{c}=\frac{3 \mu \phi Z F_{c}}{2 \pi d^{2}}
$$

where $\mu \simeq 0.4$ denotes the friction coefficient, $\phi \simeq 0.6$ is the solid volume fraction and $Z \simeq 6$ is the number of

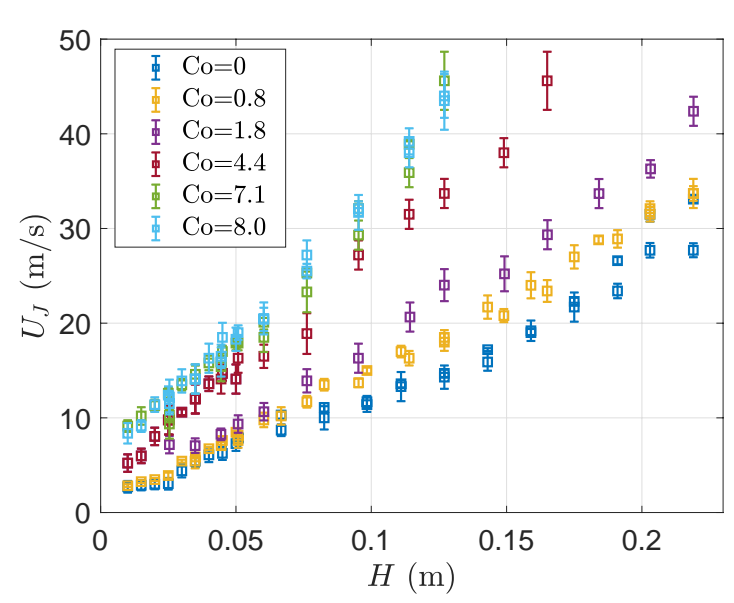

Figure 2. Mean velocity threshold of the jet at the outlet of the nozzle $U_{J}$ as a function of the distance between the nozzle and the granular bed $H$ for glass beads of diameter $d=800 \mu \mathrm{m}$ and increasing cohesive number $\mathrm{Co}=0,0.8,1.8,4.4,7.1,8.0$.

contacts per particle. The expression of the cohesive force given by Eq. (1) and the weight of a particle leads to the dimensionless parameter

$$
\mathrm{Co}=\frac{1}{9} \frac{\gamma\left(1-e^{-b / B}\right)}{\rho_{g} g d^{2}},
$$

called the cohesive number. For the grains and the range of coating thickness used here $0 \leq \mathrm{Co} \leq 8.0$. Further details and characterization are provided in Ref. [9].

\section{Measurements of the erosion threshold}

To systematically determine the erosion threshold, we initially prepare the granular bed by pouring a large quantity of grains in the container. We then flatten the surface before each experiment. We place the nozzle at a distance $H$ from the granular bed. The nozzle is turned on at a low flow rate, well below the erosion threshold. The vertical jet impacts the horizontal surface of the granular bed, and we increase the flow rate, and thus the velocity of the jet, in small increments until the first grains are eroded. The erosion threshold is then determined as the average of the last velocity where no erosion is visible and the first velocity where grains are eroded. The uncertainty on the erosion threshold measurement is the difference between these two velocities.

The threshold velocity of the jet at the outlet, $U_{J}$, at which grains start being eroded is shown in Fig. 2 for various distance to the granular bed $H$ and inter-particle cohesion. For all cohesive number, $\mathrm{Co}$, the larger the distance $H$ is, the larger the velocity of the jet $U_{J}$ needs to be so that the velocity at the surface of the granular bed is large enough to erode the grains. This observation is in agreement with the experimental results of Badr et al. obtained for cohesionless grains $[5,6]$. We also observe that for a given distance $H$ to the granular bed, the threshold velocity of the jet $U_{J}$ increases with the cohesion between the 


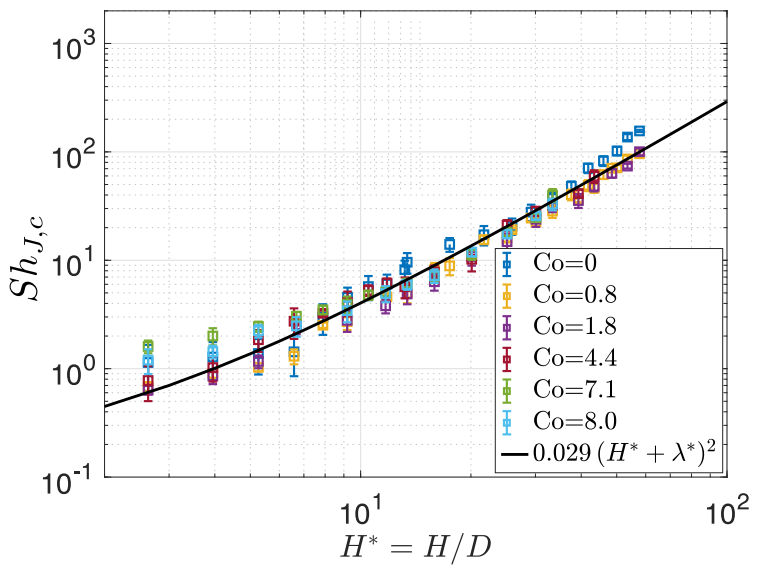

Figure 3. Global cohesive Shields number, $\mathrm{Sh}_{\mathrm{J}, \mathrm{c}}$, as a function of $H^{*}=H / D$ for the cohesion considered in Fig. 2. The solid line is given by Eq. (9).

grains. For example, when the nozzle is located at a distance $H=10 \mathrm{~cm}$ from the granular bed, the velocity of the jet required to erode the cohesive granular medium is between 2 and 3 times larger for cohesive grains $(\mathrm{Co}=8.0)$ than for non-cohesive grains $(\mathrm{Co}=0)$. Nevertheless, the global trend seems similar with and without cohesion, except a small difference when the nozzle is close to the granular bed, where the plateau value seems to disappear for cohesive grains.

\section{Cohesive Shields number}

\subsection{Global Shields number}

Two relevant dimensionless parameters are important in this situation: the dimensionless distance to the granular bed $H^{*}=H / D$ and the Shields number. We should emphasize that the Reynolds number associated with the jet is $\operatorname{Re}_{\mathrm{J}}=U_{J} D / v \geq 10^{3}$ for all experiments considered here. Therefore, the jet is turbulent, and, following the approach of Badr et al. [5], we can consider an inertial stress acting on the particles so that $\tau_{f}=\rho_{a} U_{J}^{2}$. The inertial Shields number based on the velocity of the jet at the exit of the nozzle, referred to as the global Shields number, is given by

$$
\mathrm{Sh}_{\mathrm{J}}=\frac{\rho_{a} U_{J}^{2}}{\left(\rho_{g}-\rho_{a}\right) g d}
$$

The Shields number is the ratio of the forces eroding the grain, i.e., the drag, and the forces stabilizing it. For cohesive grains, the stabilizing force is now the sum of the weight of the grains and of all inter-particle cohesion forces acting on a grain. A global cohesive Shields number can be defined as

$$
\mathrm{Sh}_{\mathrm{J}, \mathrm{c}}=\frac{F_{J}}{F_{W}+F_{c, \text { tot }}}=\frac{\mathrm{Sh}_{\mathrm{J}}}{1+F_{c, \text { tot }} / F_{W}}
$$

The resulting cohesive force acting on a grain can be estimated as

$$
F_{c, \text { tot }}=\sum_{i=1}^{N} \vec{F}_{c, i} \cdot \mathrm{d} \vec{n}_{i}=\alpha F_{c}
$$

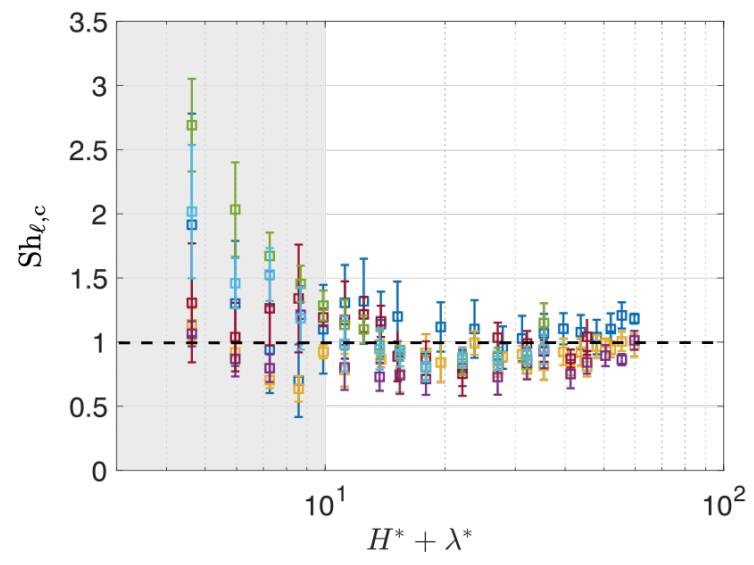

Figure 4. Local cohesive Shields number $\mathrm{Sh}_{\ell, \mathrm{c}}$ for varying interparticle cohesion. The color code is the same as in Fig. 3 and the horizontal dashed line is given by $\mathrm{Sh}_{\ell, \mathrm{c}}=1$.

where $N$ is the number of contact for one grains, $\vec{F}_{c, i}$ denotes the inter-particle cohesive force projected in the direction of the drag force, $\vec{n}_{i}$ is the local vector pointing from the center of a particle to its neighbor. The coefficient $\alpha$ is the resulting prefactor accounting for the number of cohesive contacts and the orientation of the cohesive force, so that $\alpha \sim \mu Z \phi \sum_{i=1}^{N}\left\langle\theta_{i}\right\rangle$ is expected to be of order 1 . Using the expression of the cohesive force between two grains $F_{c}=3 \pi \gamma d\left(1-e^{-b / B}\right) / 2$ and the definition of the cohesive number $\mathrm{Co}$, we then obtain a global cohesive Shields number:

$$
\mathrm{Sh}_{\mathrm{J}, \mathrm{c}}=\frac{\mathrm{Sh}_{\mathrm{J}}}{1+\alpha \mathrm{Co}}=\frac{\rho_{a} U_{J}^{2}}{\left(\rho_{g}-\rho_{a}\right) g d} \frac{1}{(1+\alpha \mathrm{Co})},
$$

where $\alpha \sim 1$.

Fig. 3 shows a rescaling of the experimental data reported previously. We observe that for all inter-particle cohesion, the results collapse on a master curve. We can also account for the increase in the global Shields number $\mathrm{Sh}_{\mathrm{J}}$ with the distance $H^{*}=H / D$. To do so, we consider a turbulent jet exiting the nozzle at the velocity $U_{J}$. The flow velocity on the axis at a dimensionless distance $x^{*}=x / D$ from the nozzle is then given by

$$
u_{0}(x)=U_{J} \frac{K_{u}}{x^{*}+\lambda^{*}}
$$

where $\lambda^{*}=\lambda / D$ is the virtual origin of the turbulent jet, and $K_{u}$ is a constant. Both parameters depend on the Reynolds number of the jet but can be considered constant at first order in the range of Reynolds number considered here. We performed experiments using cohesionless grains of different diameters and found that the dimensionless virtual origin in the present case is $\lambda^{*} \simeq 2$. Therefore, since the flow velocity at the surface of the granular bed is related through the velocity at the outlet by a factor $x^{*}+\lambda^{*}$, one expects that the global cohesive Shields number will scale as

$$
\mathrm{Sh}_{\mathrm{J}, \mathrm{c}}=\frac{C\left(H^{*}+\lambda^{*}\right)^{2}}{(1+\alpha \mathrm{Co})}
$$



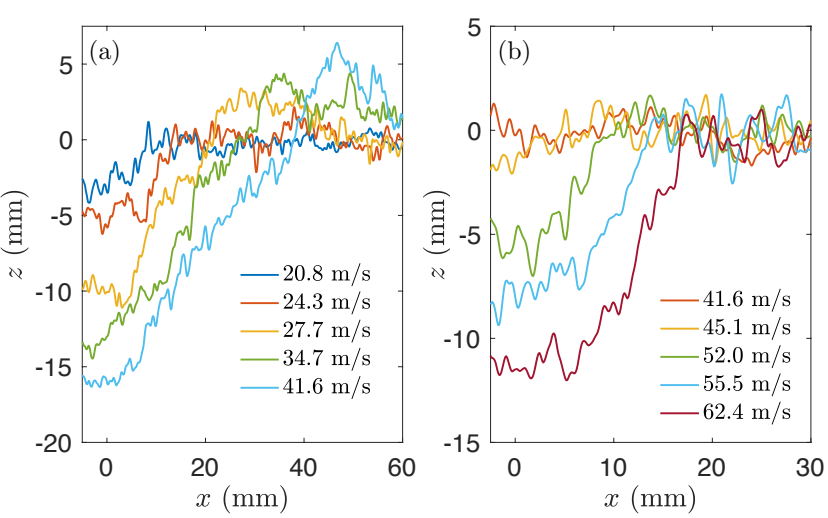

Figure 5. Morphology of the crater when increasing the velocity at the outlet of the nozzle $U_{J}$ for (a) cohesionless grains and (b) cohesive grains $(\mathrm{Co}=8.0)$.

where $C$ is an experimental constant. We observe in Fig. 3 that such scaling law captures the erosion threshold for all cohesion considered in this study. Note that this expression is valid only sufficiently far from the nozzle when the turbulent jet has adopted its self-similar profile and is thus not expected to work for $H^{*} \lesssim 10$.

\subsection{Local Shields number}

The local erosion threshold velocity must be the same for a given inter-particle cohesion whatever the distance of the nozzle to the granular bed is. Therefore, we can also build a constant local Shields number, based on the velocity in the vicinity of the granular bed. The velocity at the surface of the granular bed is given by Eq. (8). Badr et al. [5] have shown for cohesionless material that a local Shields number can be obtained through the expression

$$
\mathrm{Sh}_{\ell}=\mathrm{Sh}_{J} \frac{\left(K_{u}\right)^{2}}{\left(H^{*}+\lambda^{*}\right)^{2}}
$$

Here, we also need to take into account the inter-particle cohesive force to obtain the local cohesive Shields number. As a result, the governing parameter to characterize the erosion of cohesive particles is

$$
\operatorname{Sh}_{\ell, \mathrm{c}}=\frac{\rho_{a} U_{J}^{2}}{\left(\rho_{g}-\rho_{a}\right) g d} \frac{\left(K_{u}\right)^{2}}{(1+\alpha \mathrm{Co})\left(H^{*}+\lambda^{*}\right)^{2}}
$$

and is expected to be of order 1 . We rescaled the experimental data using this expression in Fig. 4, and we indeed observe that sufficiently far from the nozzle, typically for $H^{*} \gtrsim 10$, the experimental results for varying cohesion and nozzle-to-bed distance rescaled on a constant value $\mathrm{Sh}_{\ell, \mathrm{c}} \simeq 1$. Therefore, this dimensionless number captures both the effect of the distance between the nozzle and the granular bed, but also the inter-particle cohesive force.

\section{Conclusion}

In this study, we have shown that the erosion process of a cohesive granular material depends on the distance between the granular bed and the outlet of the nozzle and the inter-particle cohesion. The cohesive force delays the onset of erosion. However, our results also demonstrated that a local cohesive Shields number accounts for the flow associated with the turbulent jet and the cohesive force.

We also performed preliminary experiments to characterize the morphology of the crater, as can be seen in Fig. 5(a) and 5(b) for cohesionless and cohesive grains, respectively. Interestingly, whereas cohesionless grains exhibit two types of crater depending on the velocity of the jet and the distance of the nozzle to the granular bed [6], this observation does not hold for cohesive grains. Indeed, for cohesionless grains, one type of crater consists of a parabolic crater at the center and a straight slope on the edge where the grains are continuously flowing. For cohesive grains, this shape of craters is not observed since as soon as the drag force is sufficient to erode the grains, they are transported far from the crater. As can be seen in 5(b), no build-up of grains can be observed on the side of the crater for cohesive grains. This observation likely comes from the fact that the transport laws of the eroded grains are different because of the possibility to break and reorganize clusters of particles transported by the flow. Unraveling the transport process of cohesive grains is a current subject of study.

\section{References}

[1]K. J. Berger, A. Anand, P. T. Metzger, C. M. Hrenya, Phys. Rev. E 87, 022205 (2013)

[2]G. J. Hanson, K. R. Cook, Appl. Eng. Agric. 20, 455 (2004)

[3]F. Lachaussée, Y. Bertho, C. Morize, A. Sauret, P. Gondret. Phys. Rev. Fluids 3, 012302 (2018)

[4]J. J. Hastings, D. Rhodes, A. S. Fellerman, D. McKendrick, C. Dixon, Powder Technol. 174, 18-24 (2007)

[5]S. Badr, G. Gauthier, P. Gondret, Phys. Fluids 26, 023302 (2014)

[6] S. Badr, G. Gauthier, P. Gondret, Phys. Fluids 28, 033305 (2016)

[7]F. Brunier-Coulin, P. Cuellar, P. Philippe, Phys. Rev. Fluids 2, 034302 (2017)

[8]F. Brunier-Coulin, P. Cuellar, P. Philippe, Phys. Rev. Fluids 5, 034308 (2020)

[9]A. Gans, O. Pouliquen, M. Nicolas, Phys. Rev. E 101, 032904 (2020) 